\title{
Nootropic, Neuroprotective and Anti-oxidant Role of Apocynin in Scopolamine Induced Memory Deficit in a Zebrafish Model
}

\author{
Sneha Bagle, Suraj Muke, Vaibhavi Peshattiwar, Aakruti Kaikini and Sadhana Sathaye* \\ Institute of Chemical Technology, Nathalal Parekh Marg, Matunga, Mumbai-400019, India
}

${ }^{*}$ Corresponding author: Prof. Sadhana Sathaye, Institute of Chemical Technology, Nathalal Parekh Marg, Matunga, Mumbai-400019, India; Tel: +91-22 33612218; Email: sadhanasathaye@hotmail.com

Received: March 25, 2021; Accepted: April 05, 2021; Published: April 12, 2021

\begin{abstract}
Four decades of search in quest of an effective therapeutic option for Alzheimer's disease (AD) has led to a complete clinical failure, putting pre-clinical models, their evaluation in spotlight. The pre-clinical stage if lead with a systemic approach, than jumping straight into clinical trials, will reduce financial burden opening avenues for effective AD therapeutics. Preliminary screening therefore shall be fast, ensuring use of efficient, easy, and less time consuming pre-clinical models for screening new entities. Aim of present research was therefore development of a quick, effective, and easy zebrafish model for preliminary screening of probable AD therapeutics. The present research used total of 72 zebrafish divided into six groups, control, negative control, vehicle control, and 3 groups for apocynin $(10 \mathrm{mg} / \mathrm{kg}, 30 \mathrm{mg} / \mathrm{kg}$, and $70 \mathrm{mg} / \mathrm{kg})$. We investigated memory retention, long term memory retention using passive avoidance paradigm, free radical generation using DCFDA assay, and glial cell proliferation using $\mathrm{H}$ and $\mathrm{E}$ histological staining. Intraperitoneally administered scopolamine $(0.025 \mathrm{mg} / \mathrm{kg})$ induced memory deficits hindering memory formation. Apocynin at all doses prevented scopolamine-induced amnesia. Apocynin was found to be capable of reducing free radical load and curbing the inflammation due to scopolamine administration. Moreover; long term memory retention was observed with $10 \mathrm{mg} / \mathrm{kg}$ apocynin. Our revelations designate the fact that passive avoidance model in zebrafish could be used as an acute model in order to screen potential entities for treatment of AD. Our findings highlight that a single dose of apocynin could reverse memory deficits induced by single scopolamine injection by acting on cholinergic transmission pathway. Apocynin being an anti-oxidant reduced the free radical generation, thus engaging one of the crucial pathways toward AD pathophysiology. In addition it offered neuroprotection by reducing glial cell proliferation. In conclusion apocynin exhibited multifaceted pharmacological activities like being nootropic, neuroprotective, and anti-oxidant in preclinical AD model.
\end{abstract}

Keywords: Alzheimer's disease (AD); Scopolamine; Passive avoidance; Apocynin; Zebrafish; Nootropic; Neuroprotective

\section{Introduction}

The cost of bringing a medicine from invention to pharmacy shelves is $\$ 2.8$ billion [1]. A lot of molecules are failing miserably in the clinical phase of AD. Therefore a systematic approach in the initial drug discovery process is of utmost importance. A number of models are being developed to mimic the $\mathrm{AD}$ pathogenesis and the existing ones are being fine tuned to meet the demand. To curtail down the search for a therapeutic option from a humongous number i.e. to generate a lead from various hits will require use of an efficient, fast, and effective in-vivo pre-clinical model. Zebrafish as a pre-clinical model is equipped with all these characteristics.

The zebrafish (Danio rerio) is fast emerging as a promising model organism to study various central nervous system (CNS) disorders, including $\mathrm{AD}$ [2]. It has recently become a focus in neurobehavioral studies since it displays quantifiable neuropathological and behavioural phenotypes [3,4]. Zebrafish have a fully characterized genome, and exhibit significant physiological homology to mammals, including humans [5]. Zebrafish is an ideal model for the study of human diseases because they present a number of features that make it unique as an animal model. Zebrafish brain is highly conserved of the basic brain organization, with similar key neuroanatomical and neurochemical pathways of relevance to human diseases [6,7]. In the recent years zebrafish has been proposed as a valid experimental paradigm to study AD [8]. Scopolamine (SCP) is a well known and extensively studied muscarinic cholinergic antagonist which has several effects in central and peripheral nervous system $[9,10]$. Both clinical $[11,12]$ and rodent $[13,14]$ studies point towards its ability to induce cognitive deficits. Substantial evidences also indicate the potential of acute scopolamine to develop memory deficits in zebrafish [15-20]. Current research strategies target the unused potential of phytoactives and marine actives as therapeutic moieties in AD. Apocynin an inhibitor of NADPH oxidase is a naturally occurring methoxy-substituted catechol [21]. It is isolated from the roots of Apocynin cannabinum (Canadianhemp), Picrorhizakurroa (Scrophulariaceae) and Jatropha multifia (Euphorbiaceae) [22-24]. It is known to possess various biological activities like anti-inflammatory, anti-oxidant and is useful in many disorders such as neurodegeneration, asthma, cancer, arteriosclerosis, hypertension etc. [21]. Apocynin was found to be effective in 1-methyl-4-phenyl-1, 2, 3, 6-tetrahydropyridine (MPTP) 
marmoset model. It compensated for the loss of dopamine, mitigated the parkinsonian signs, symptoms, and improved motor function [25]. All this literature encouraged us to investigate apocynin in zebrafish passive avoidance paradigm as a model for $\mathrm{AD}$. In the current research we demonstrate the development of an acute zebrafish model which is easy, quick, and useful for screening new potential entities for $\mathrm{AD}$, to arrive at possible lead in the drug discovery process. We have also explored the passive avoidance paradigm to study the memory deficits induced by a single scopolamine administration and its subsequent reversal by apocynin.

\section{Materials and Methods}

\section{Reagents and Chemicals}

Apocynin, (-)-Scopolamine hydrobromide trihydrate, 2', 7'-dichlorofluorescin diacetate (DCF-DA) procured from sigma Aldrich, USA, Tween 80 from S D Fine Chemicals ltd, India. All other chemicals used were of analytical grade.

\section{Animals}

Indigenous transgenic male adult zebra fish strain Danio rerio (3-4 months old) were used for this study (obtained from Dolphin Aquaculture, Mumbai, India). The fish chosen were of uniform size in length $(3 \mathrm{~cm} \pm 0.5 \mathrm{~cm})$ and weight $(500 \pm 50 \mathrm{mg})$. They were maintained in 25 litres glass storage tank containing purified dechlorinated water to neutralize heavy metals, chlorine, and chloramines present in the water that could be harmful to fish. Fish tank temperature was maintained at $25^{\circ} \mathrm{C} \pm 2^{\circ} \mathrm{C}$ under a 12:12-hr light: dark cycle and were continuously aerated. Fish were fed thrice daily with brine shrimp (marketed as Instincts ${ }^{\circledR}$ ). The temperature, oxygen, and water quality was maintained throughout the experimental procedures and the fishes were allowed to acclimatize to the laboratory conditions for 9 days [26]. All solutions administered were prepared fresh at room temperature.

\section{Procedure}

The dose for scopolamine administration was chosen based on previous literature [27]. Scopolamine was administered intraperitoneally in zebrafish after the training sessions using $6 \mathrm{~mm}$ X 31 G needle attached to $26-\mathrm{G} 10 \mu \mathrm{l}$ Hamilton syringe with help of a catheter of size 24-G using Kinkel MD protocol with modifications [28]. Briefly, the zebra fish were cryoanesthetized prior to intra peritoneal injection until the fish shows reduced respiration and lack of motor coordination. The anesthetized fish was gently put in a water-soaked sponge with the abdomen facing up and the head of the fish positioned at the hinge of the sponge. The needle was inserted into the midline of the abdomen parallel to the spine and posterior to the pectoral fins. The procedure was mastered to complete the injection within $10 \mathrm{sec}$. Following injection, the fishes were kept in separate tanks to facilitate the recovery post anaesthesia with water maintained at $25 \pm$ $2^{\circ} \mathrm{C}$ along with proper aeration. Apocynin was administered orally. In order to guarantee the doses administered, apocynin was suspended in Tween 80 (1\%) in distilled water. Apocynin was administered orally after 1 hour of scopolamine administration using $24-\mathrm{G}$ catheter fitted into the hypodermic needle of 26-G $10 \mu \mathrm{l}$ Hamilton Syringe using
Collymore C protocol with modifications [29]. Briefly, the fish was cryoanesthetized and held in water soaked sponge, in such a way that the fish was held vertically upward with its back positioned in the hinge of the sponge. The flexible tubing, for oral gavage was lowered into the oral cavity of the fish, approximately $1 \mathrm{~cm}$, until the tip of the tubing extended past the gills. The drug solution was then injected slowly into the intestinal tract. The volume of drug administered was $2 \mu$ l. The injection procedure was mastered to complete the injection procedure within $10 \mathrm{sec}$. Following injection, the fishes were kept in a separate tank to facilitate the recovery post anaesthesia, with water maintained at $25 \pm 2^{\circ} \mathrm{C}$ along with proper aeration. After every administration the fishes were transferred into a new clean tank. Tween 80 was used as the vehicle. The vehicle, scopolamine, and apocynin were prepared freshly on the experimental day. Post injection and oral administration fishes which did not recover within 2 to 3 min were excluded from the experiment. The fishes were divided into six groups, namely control, negative (SCP $0.0025 \mathrm{mg} / \mathrm{kg}$ ip), vehicle (tween 80 po), apocynin 3 groups; APO $10 \mathrm{mg} / \mathrm{kg}$ po, APO $30 \mathrm{mg} / \mathrm{kg}$ po, APO $70 \mathrm{mg} / \mathrm{kg}$ po; with 12 fishes in each group.

\section{Passive Avoidance Experimental Chamber}

Individually zebrafish were trained and tested for the long-term memory retention in the passive avoidance task. The apparatus consists of $18 \mathrm{~cm}$ length $\times 9 \mathrm{~cm}$ width $\times 7 \mathrm{~cm}$ height glass tank divided in two equal half compartments designated as dark and white (differentiated by the colour of the tank), by a manually operated sliding door $(9 \mathrm{~cm}$ $\times 7 \mathrm{~cm}$ ). The tank water level was maintained at three $\mathrm{cm}$ and the partition was raised one $\mathrm{cm}$ above the tank floor to allow zebrafish to swim freely from one side to the other $[15,16,30]$.

\section{Passive Avoidance Paradigm}

On training session, fish was placed in the white side of the tank with the partition between compartments being closed. The fish was allowed to acclimatize in it for three min and the partition was then raised till one $\mathrm{cm}$ height. The fish was given five min to enter the dark compartment through this partition. The partition door was closed once the fish entered the dark compartment completely with its entire body. A marble was dropped in front of the fish head; three sec after it entered the dark compartment. The fish was then immediately removed from the dark compartment and placed in a separate tank. This whole procedure is considered as one training session. The marble dropped in front of the fish served as shock for the fish, so that it would be reluctant to enter the dark compartment. Each fish was trained thrice with an interval of three min between each training session $[15,16,30]$. Each training session hereafter will be addressed as a trial. After the three trial session, a test session was conducted after two hr of the last trial (documented as day 1) for control group fishes. The test session consisted of repeating of the trial session except, the marble was not dropped when the fish entered the dark compartment. For negative control group, SCP was administered ip after $1 \mathrm{hr}$ of the last trial. The test session was conducted one hr post SCP administration, documented as day 1 . For the vehicle and test drug groups, the vehicle ( $1 \%$ tween 80 ) or the test drug (apocynin $10 \mathrm{mg} / \mathrm{kg}, 30 \mathrm{mg} / \mathrm{kg}$, and 70 $\mathrm{mg} / \mathrm{kg}$ ) were administered per oral one hr after SCP administration. 
The test session was conducted one hr after the vehicle or test drug administration, documented as day 1 . For all the groups only the test session was repeated after $24 \mathrm{hrs}$, documented as day 2; and after 48 hrs documented as day 3 after the first test session (day 1); to assess the long term memory retention. The flowchart for the passive avoidance test for all the groups is depicted in Figure 1. The latency to completely enter the dark compartment was measured for the three trials and the three test sessions; used as an index of memory retention.

\section{Reactive Oxygen Species Formation}

The fishes in all the groups, after $48 \mathrm{hrs}$ or after the test 3 of the study were subjected to cryoanaesthesia, according to the NIH guidelines. The fishes were first immobilised, by keeping them in ice water [5:1 (ice: water)] for at least 10 minutes or until cessation of opercular movement. The fishes were left for 20 minutes in ice cold water until cessation of all movements. After the fishes were euthanized, brains were isolated. The brains were homogenized with equal volume of ice-cold 0.1 $\mathrm{M}$ phosphate buffer saline ( $\mathrm{pH}$ 7.4). The homogenate was then centrifuged at $4^{\circ} \mathrm{C}(10,000 \mathrm{rpm}$; R-248Mof CPR-24 plus Instrument, Remi, India) for $15 \mathrm{~min}$, and aliquots of homogenate were used for estimation of free radicals. The total concentration of free radicals, especially reactive oxygen species, was detected using 2', 7'-dichlorofluorescin diacetate (DCFDA) with slight modification of Pereira AG et al. protocol [31]. The $30 \mu \mathrm{l}$ brain homogenate was treated with $100 \mu \mathrm{l}$ of $100 \mu \mathrm{M}$ DCFDA in 96 well plate. The plate was incubated at $37^{\circ} \mathrm{C}$ for $30 \mathrm{~min}$ in the dark. The whole protocol was performed in dark. The formation of the oxidized fluorescent derivative dichlorofluorescein (DCF) was monitored at 488 $\mathrm{nm}$ excitation and $525 \mathrm{~nm}$ emission using a fluorescence microplate spectrophotometer (Epoch, Biotech, USA).

\section{Histopathological Analysis}

After the completion of the inhibitory avoidance task, fishes were subjected to cryoanaesthesia. The brain was then isolated and stored in $4 \%$ paraformaldehyde solution until histopathological analysis using hematoxylin-eosin ( $\mathrm{H}$ and $\mathrm{E}$ ) staining for evaluation of neuronal damage by examining the glial cell proliferation. The stained sections were evaluated by a histopathologist unaware of the treatments.

\section{Statistical Analysis}

Inhibitory avoidance memory data are presented as mean \pm S.E.M. Statistical analyses were performed using GraphPad Prism 5 software for Windows (GraphPad Software, San Diego, CA, USA). Latencies of individual group and between multiple groups were compared using one way ANOVA followed by Newman-Keuls test (post-hoc analysis). The level of significance was set as \#\#\#P<0.001 when compared with control group, ${ }^{* *} \mathrm{P}<0.001$ when compared with scopolamine treatment group. The inter day latencies between multiple groups were compared using Two-way ANOVA followed by Bonferroni test as a post-ANOVA test. The level of significance was set as $\# \mathrm{P}<0.05$ when compared with control group, ${ }^{\star} \mathrm{P}<0.05$ when compared with scopolamine treatment group. The generation of free radical was analysed using one-way ANOVA followed by Post hoc Dunnett's test. The level of significance was set as \#\#\# $\mathrm{P}<0.001$ when compared with control group and ${ }^{* * *} \mathrm{P}<0.001$ when compared with scopolamine treatment group.

\section{Results}

\section{Effect of SCP on Zebrafish in Passive Avoidance Paradigm}

The effectiveness of scopolamine in altering the memory was evaluated in the inhibitory avoidance task, by training the fishes in 3 consecutive trials followed by a test session. As evident in Figure 2b, the test latency for SCP decreased significantly compared to the third trial in the SCP group $(\mathrm{P}<0.001)$, whereas the test latency significantly

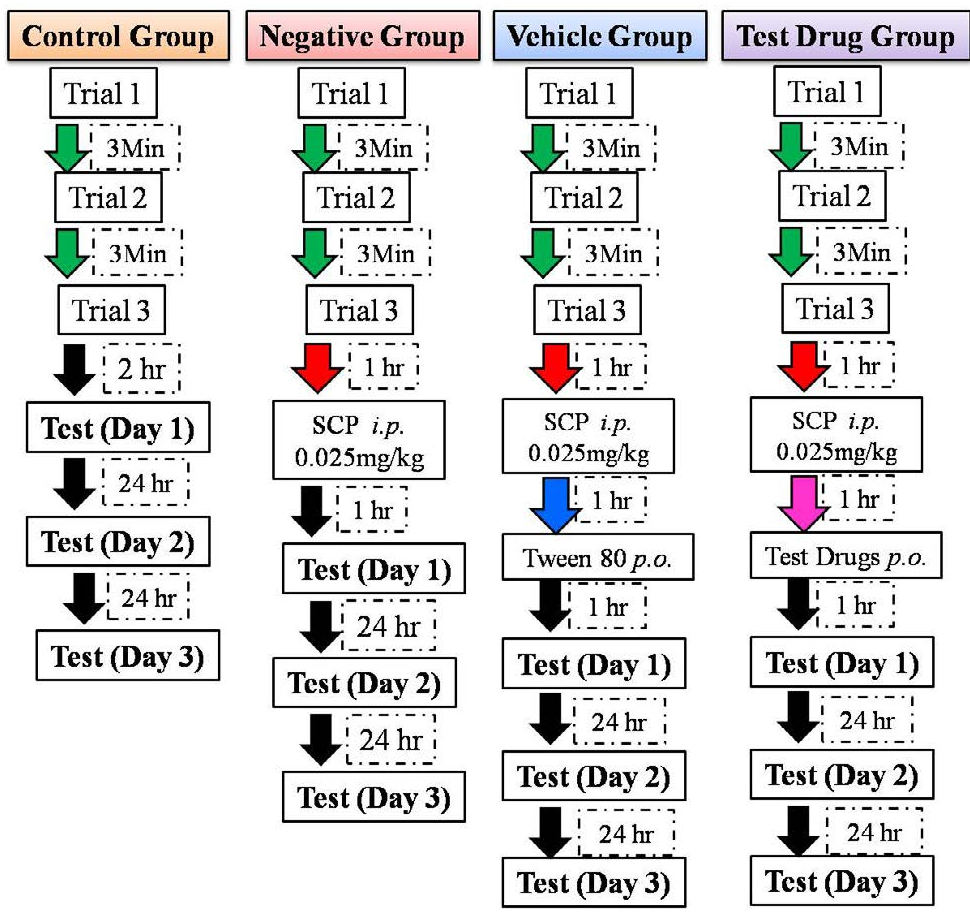

Figure 1: Flowchart for passive avoidance test followed for all the groups. 


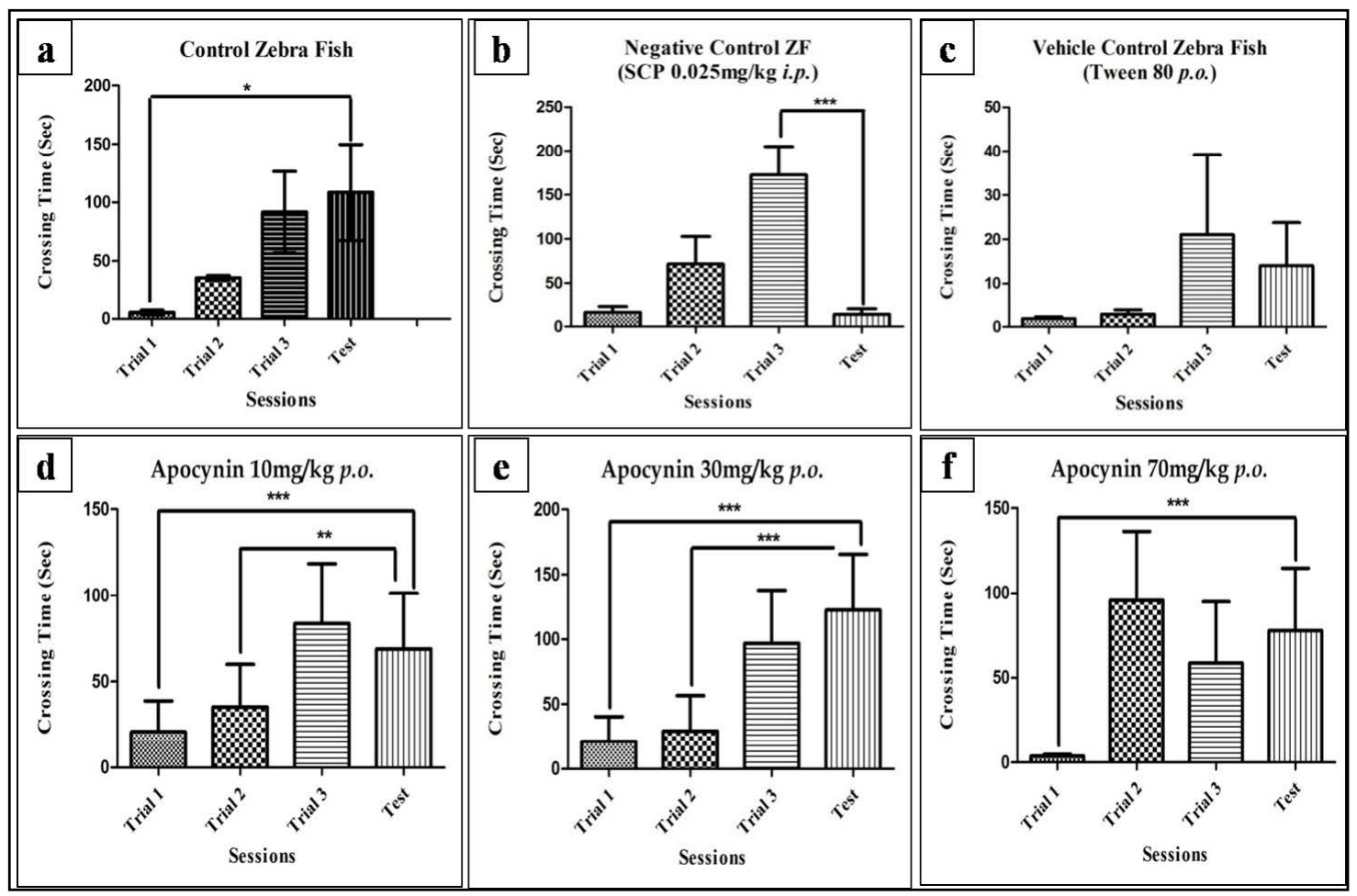

Figure 2: Individual training session of fishes in control (a), negative control (b), vehicle control (c), AL (d), AM (e) and AH (f) group on day 1 . Data expressed as mean \pm SEM. ${ }^{\star} \mathrm{P}<0.05$, ${ }^{* *} \mathrm{P}<0.01$, and ${ }^{* * *} \mathrm{P}<0.001$ when compared to the test using One-way ANOVA followed by Post hoc Student - Newman Keuls Test ( $\left.\mathrm{n} \sim 12\right)$.

increased in the control group compared to the first trial $(\mathrm{P}<0.05)$, evident in Figure 2a. The test latency for the vehicle control group also decreased when compared to the third trial, though the decrease was not significant (Figure 2c).

\section{Effect of Apocynin on Zebrafish in Passive Avoidance Paradigm}

Apocynin at all three doses was able to reverse the SCP induced memory deficits. The test latency for apocynin at $10 \mathrm{mg} / \mathrm{kg}$ was significantly higher than trial 1 with $\mathrm{P}<0.001$ and trial 2 , with $\mathrm{P}<0.01$ (Figure 2d). The test latency for apocynin at $30 \mathrm{mg} / \mathrm{kg}$ was significantly higher than trial 1 and trial 2, with $\mathrm{P}<0.001$ for both (Figure 2e). However, for $70 \mathrm{mg} / \mathrm{kg}$ the test latency was significantly higher than the first trial, with $\mathrm{P}<0.001$ (Figure 2f).

The inter group test latencies were also compared and are depicted in Figure 3. SCP significantly reduced the escape latency when compared to the control group latency with $\mathrm{P}<0.001$. Apocynin significantly increased the test latencies at all three doses when compared with the SCP group, with $\mathrm{P}<0.001$ at all doses. The escape latency in the vehicle control group was reduced when compared to the latency for control group, but the decrease was not significant.

\section{Long-Term Memory Retention}

Our intent was to explore the effect of single dose administration of both scopolamine and the test entity apocynin on long term memory retention of the learned response in passive avoidance paradigm. The results are depicted in Figure 4. On day one the fishes in control group showed the highest escape latency, the observation being persistent for next two days (day 2 and 3) as well. The negative control group

\section{Passive Avoidance Test in Zebra Fish Apocynin}

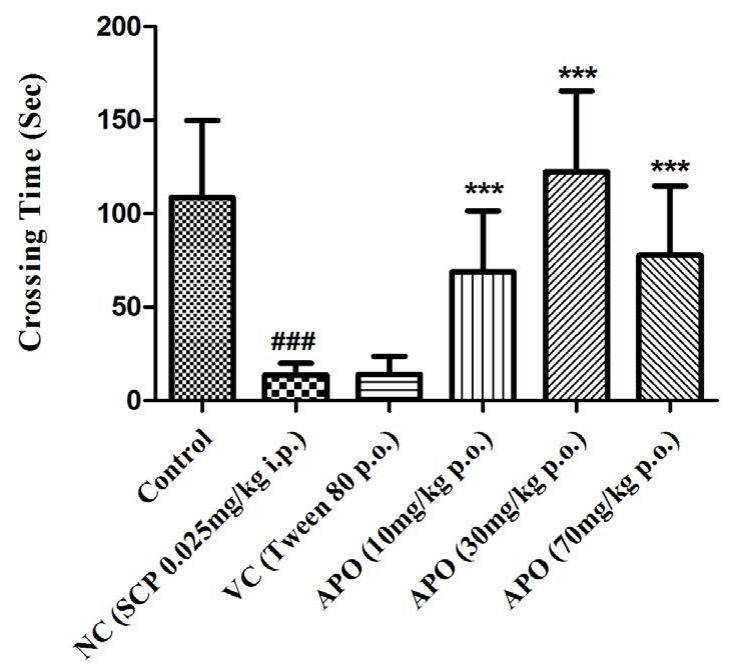

Figure 3: Comparison of day 1 test latencies between all the groups with respect to apocynin. Data expressed as mean \pm SEM. \#\#\#P<0.001, when compared with Control group, ${ }^{* * *} \mathrm{P}<0.001$ when compared with Negative Control group using one-way ANOVA followed by Post hoc Student -Newman Keuls Test ( $\mathrm{n} 12)$.

showed lower escape latencies for all three days, with $\mathrm{P}<0.05$ for day one when compared to the control group. The vehicle control group also showed lower escape latency for all three days, but the results were not significant. Apocynin at all three doses showed improved escape latencies compared to the SCP group for day one and day two. Apocynin at $30 \mathrm{mg} / \mathrm{kg}$ showed significantly higher escape latency with $\mathrm{P}<0.05$ on day 1 . On day three the dose of $10 \mathrm{mg} / \mathrm{kg}$ showed betterment in the 


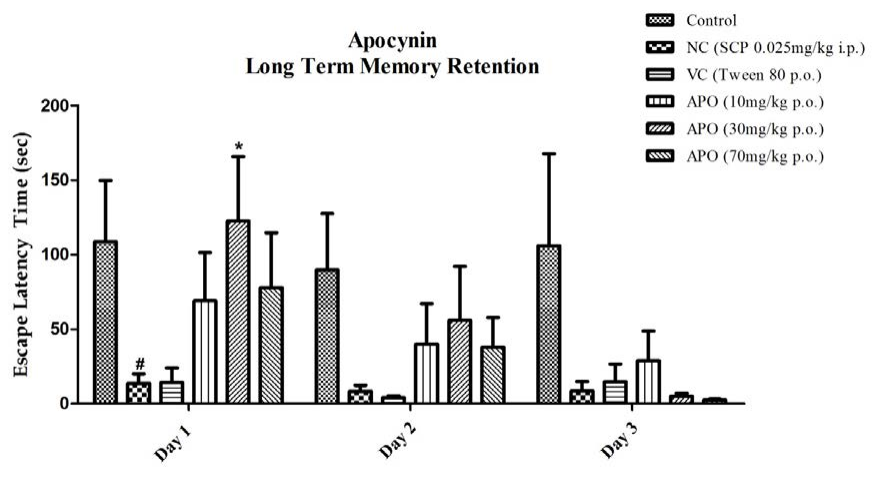

Figure 4: Inter day comparison between all groups with respect to apocynin. Data expressed as mean \pm SEM. $\# \mathrm{P}<0.05$ when compared with Control group and ${ }^{*} \mathrm{P}<0.05$ when compared to the Negative Control group. Data analyzed by using Two-way ANOVA followed by Bonferroni test as a post-ANOVA test.

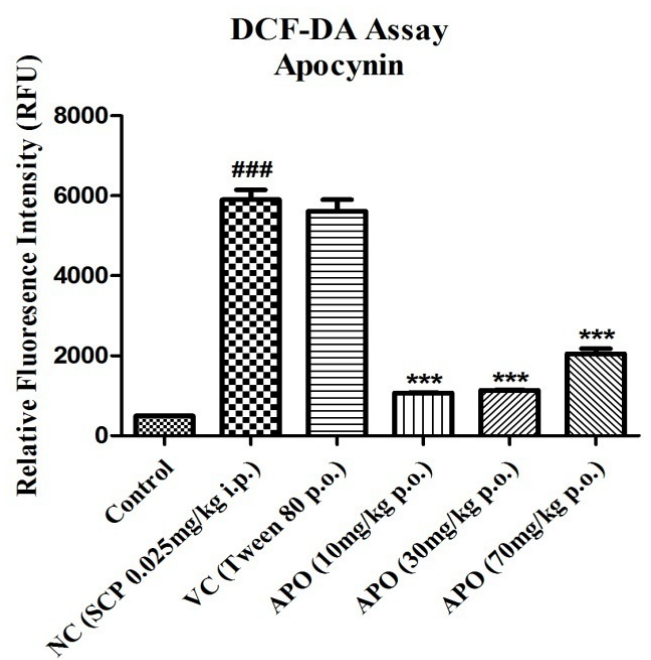

Figure 5: Effect on levels of ROS (directly proportional to RFU) in different experimental groups. Data expressed as mean $\pm \mathrm{SEM} \# \# \# \mathrm{P}<0.001$ compared with Control group and ${ }^{* * *} \mathrm{P}<0.001$ compared with Negative Control group using one-way ANOVA followed by Post hoc Dunnett's test $(\mathrm{n}=2)$. latency amongst the three doses, although not significant.

\section{Free Radical Generation (DCF-DA Assay)}

The effect of SCP administration on the generation of free radicals was assessed using the DCF-DA assay and depicted in Figure 5. SCP administration significantly increased the relative fluorescence intensity with $\mathrm{P}<0.001$ when compared with the fluorescence intensities in control group. A similar increase is seen in vehicle control group, though not significant. Apocynin at all three doses alleviated the fluorescence intensity significantly with $\mathrm{P}<0.001$ for all doses when compared with the SCP group.

\section{Histopathological Analysis (H and E Staining)}

The effect of SCP treatment on the inflammatory response was studied and the histopathological changes observed in the zebrafish brain have been presented in Figures 6 and 7. The control group did not showcase any glial cell proliferation with the score being zero. The negative control and vehicle control group exhibited significant glial cell proliferation with a score of +++ , being the severe damage depicted in Figure 6 (Control, NC, VC) and scored in Table 1. Apocynin at dose of $10 \mathrm{mg} / \mathrm{kg}$, and $30 \mathrm{mg} / \mathrm{kg}$ (Figure $7 \mathrm{AL}, \mathrm{AM}$ ) showed zero or nil glial cell proliferation. Apocynin at $70 \mathrm{mg} / \mathrm{kg}$ (Figure $7 \mathrm{AH}$ ) showcased the score of ++ denoted as moderate change.

\section{Discussion}

Table 1: Histopathological scoring for zebra fish brain images

\begin{tabular}{|l|c|}
\hline Group & Scoring \\
\hline Control & Nil \\
\hline Negative Control (NC) & +++ \\
\hline Vehicle Control $(\mathrm{VC})$ & +++ \\
\hline Apocynin $10 \mathrm{mg} / \mathrm{kg}(\mathrm{AL})$ & 0 \\
\hline Apocynin $30 \mathrm{mg} / \mathrm{kg}(\mathrm{AM})$ & 0 \\
\hline Apocynin $70 \mathrm{mg} / \mathrm{kg}(\mathrm{AH})$ & ++ \\
\hline
\end{tabular}

Note: - += Mild Change; $++=$ Moderate change/Damage; $+++=$ Severe change $/$ Damage

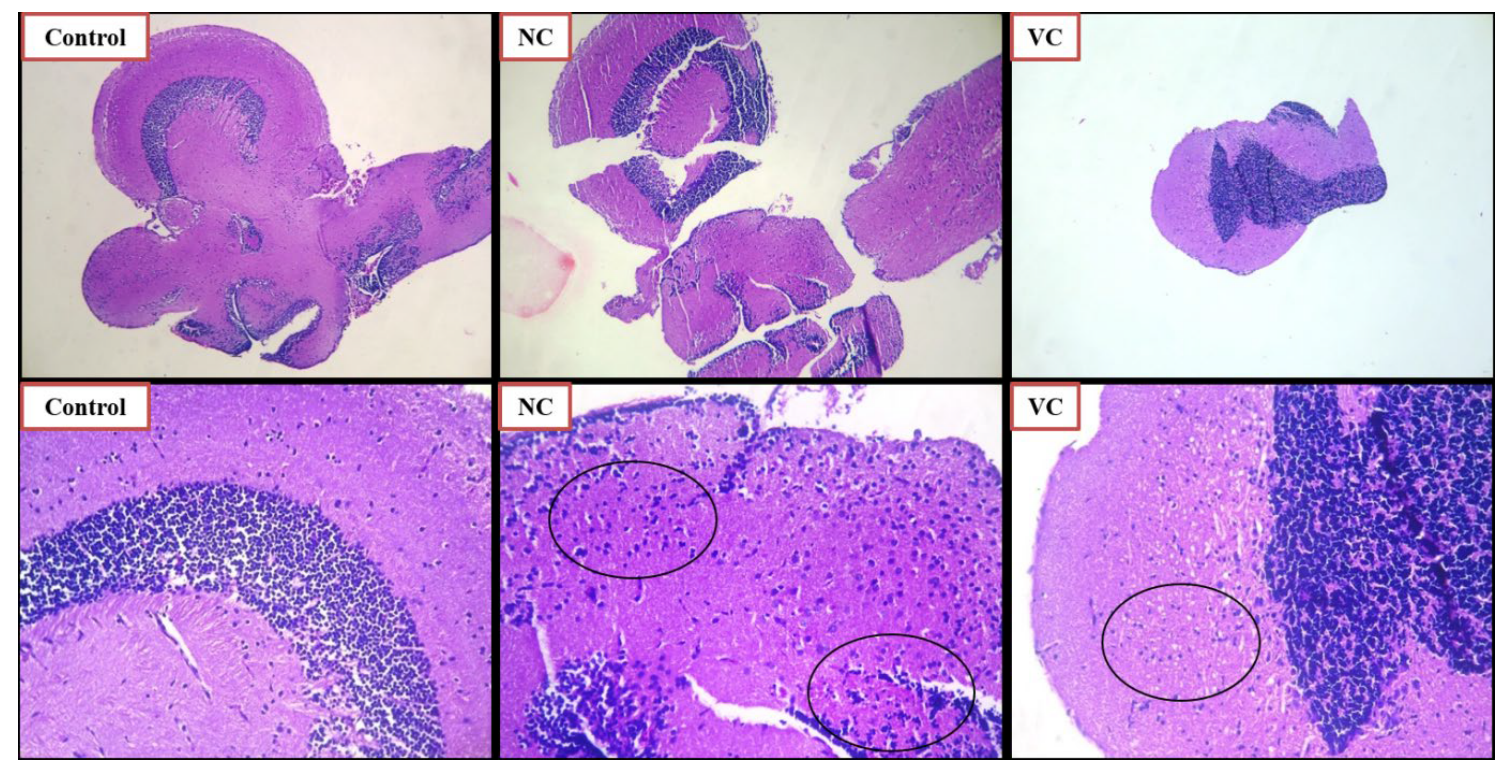

Figure 6: Histopathological analysis using H and E staining of zebrafish brains for control, negative control SCP $0.025 \mathrm{mg} / \mathrm{kg}$ (NC) and vehicle control (VC) group. The lower row indicates magnified images of the images in the above row, at magnification of 100X. The black circles indicate the glial cell proliferation. 


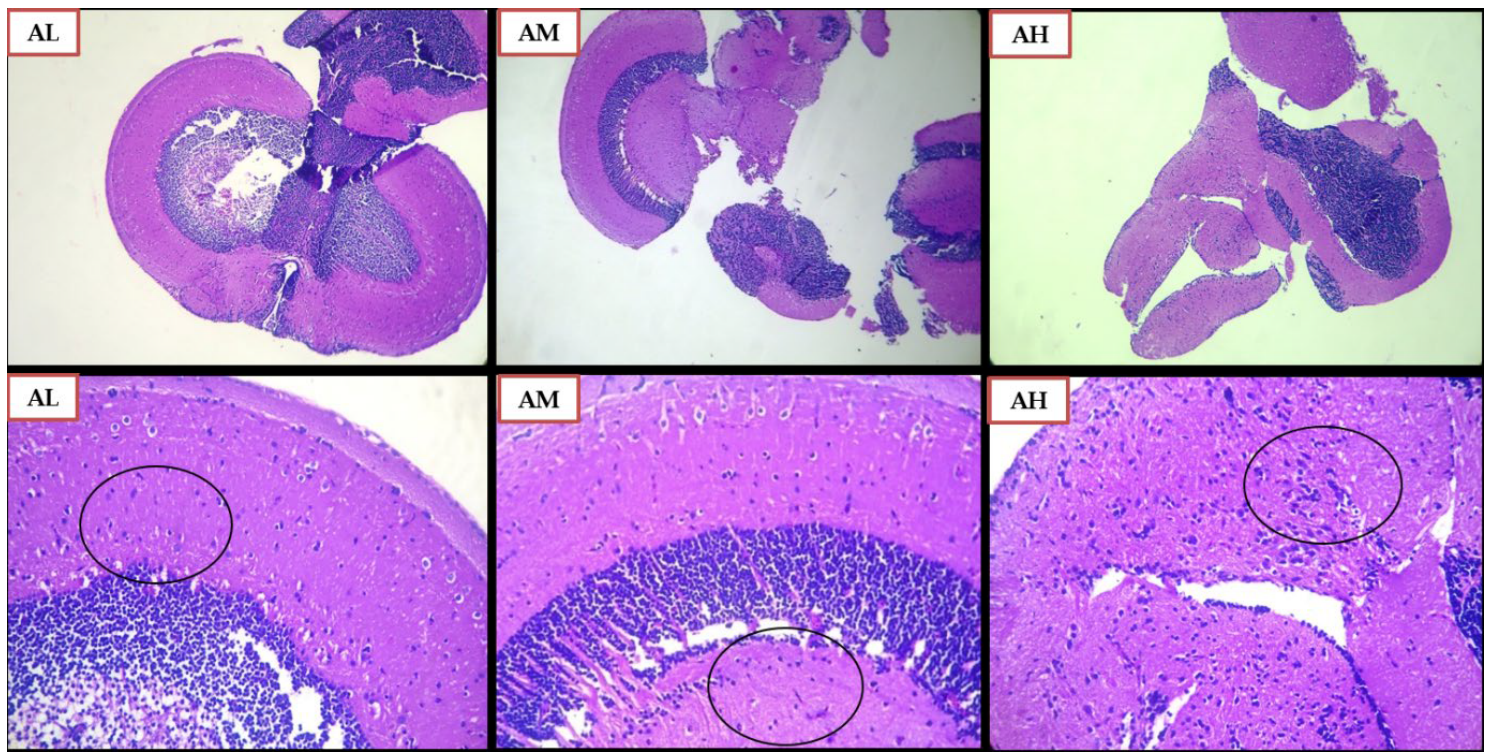

Figure 7: Histopathological Analysis using H and E staining of zebrafish brains for Apocynin Low dose $10 \mathrm{mg} / \mathrm{kg}$ (AL), Apocynin Medium dose $30 \mathrm{mg} / \mathrm{kg}$ (AM) and Apocynin High dose 70 $\mathrm{mg} / \mathrm{kg}(\mathrm{AH})$ group. The lower row indicates magnified images of the images in the above row, at magnification of $100 \mathrm{X}$. The black circles indicate the glial cell proliferation.

Scopolamine, a non-selective muscarinic receptor antagonist induces learning deficits in zebrafish is a well-established fact [30]. It is a centrally acting anti-cholinergic involved in learning and memory impairment; particularly short-term memory formation [32,33]. Therefore; the memory deficits due to scopolamine are regarded as cholinergic deficit or 'cholinergic amnesia' [34]. Also, zebra fish has been widely explored as an animal model in AD. In our study, we examined the above facts for screening apocynin in a passive avoidance paradigm. Our results demonstrate that scopolamine hampers the learning process as the latency to cross the partition of the sliding door reduced significantly in the negative control group as seen in the individual training result as compared to the control group. Our data is in accordance with the available literature on rodents and supports scopolamine's effect in evaluating memory enhancing drugs in zebrafish model. The SCP administration has induced memory deficits as evident with the decreased escape latencies. Apocynin was capable of ameliorating the effects of SCP, showcased by the comparison of test latencies for day one. The effect for apocynin however was not dose dependent as at $70 \mathrm{mg} / \mathrm{kg}$, decrease in the escape latency is observed. This may attributed to the ceiling effect at $30 \mathrm{mg} / \mathrm{kg}$. A decrease in the test latency for the vehicle control group is also observed. In the vehicle control group, the vehicle (tween 80) was administered after SCP administration, thus the decreased test latency demonstrates the effect of SCP that is responsible for the decrease in latency. Tween 80 itself does not impart any pharmacological effect in reversing the memory deficits. It merely acts a vehicle for administering apocynin. The effect of SCP administration persisted for $48 \mathrm{hrs}$ indicating the deficit in memory retention due to single dose of SCP as seen in the negative control group. Since we wanted to explore the effect of single dose administration of scopolamine and apocynin on long term memory retention, we did not administer the drug on daily basis or for 2 consecutive days. Also the training sessions of three trials was not repeated on day 2 or day 3 , only the test. Apocynin mitigated the effect of SCP and was capable of maintaining the effect for 24 hrs (day 2) and 48 hrs (day 3) for $10 \mathrm{mg} / \mathrm{kg}$. SCP causes memory impairments by affecting cholinergic transmission; apocynin reversed the memory deficit implying that it works on the cholinergic neurotransmission pathway $[34,35]$. Therefore; apocynin has a potential as an anti-Alzheimer drug and can be explored further in a rodent model of $\mathrm{AD}$. There is ocean of evidence entailing that oxidative damage induced due to free radical may play a role in $\mathrm{AD}$ pathogenesis. SCP administration generates free radicals and damages the anti-oxidant defence mechanism by damaging the anti-oxidant enzymes [36]. This fact is well established in rodent models [37] as well as in zebrafish [38]. Our study demonstrates that even single dose of scopolamine significantly induces the generation of free radicals. Apocynin being an anti-oxidant reduces the free radical generation [39]. This reduction however was not dose dependent, the $70 \mathrm{mg} / \mathrm{kg}$ dose showed a slight increase in the fluorescence intensity compare to the $30 \mathrm{mg} / \mathrm{kg}$ dose. This may be due to the ceiling effect at the 30 $\mathrm{mg} / \mathrm{kg}$ dose. The reduction in free radical formation has undeniably ameliorated the associated damage to the neuronal environment. Thus apocynin could help in halting the disease progression by alleviating the free radical production.

Glial cells are one of the most abundant cells of the brain, performing various biological functions and play a major role in inflammation. In acute phase after injury inflammation is tightly controlled and is a normal and a necessary process maintaining the homeostasis. However, in chronic phase it is detrimental, as seen in AD. It may not be the initiating factor but it significantly contributes to disease progression. Neuroinflammation induces a complex and dynamic change in glial cell phenotypes. Microglial cells are the first cell types to respond post injury they retract their processes and migrate towards the site of injury, where they release pro-inflammatory cytokines such as interleukin-1 $\beta$ (IL-1 $\beta$ ), tumor necrosis factor- $\alpha$ (TNF- $\alpha$ ), and IL-6 [40]. The histopathological data attests the role of SCP in setting up an inflammatory response capable 
of damaging the neuronal environment. Administration of single dose of SCP demonstrates the highest score for glial cell proliferation and hence the severe neuronal damage. The damage was totally reversed by apocynin $10 \mathrm{mg} / \mathrm{kg}$ and $30 \mathrm{mg} / \mathrm{kg}$ as evident with nil glial cell proliferation. The other dose of apocynin $70 \mathrm{mg} / \mathrm{kg}$ was effective compared to the negative control group, but could not completely reverse the damage. Apocynin thus bestowed neuroprotection by mitigating the neuronal damage, ultimately maintaining the neuronal homeostasis against the mulct of scopolamine administration. Our study reveals and advocates that passive avoidance test in zebrafish can be used as an acute preliminary in-vivo model to screen potential anti-Alzheimer entities. Our findings emphasize that the phytoactive apocynin reversed the memory deficits and retained the memory hampered by scopolamine administration. Apocynin thus is a nootropic with the probable mechanism being through the action on cholinergic transmission pathway. Apocynin being an anti-oxidant reduced the free radical generation, thus engaging one of the crucial pathways toward AD pathophysiology. Additionally it conferred neuroprotection by reducing the glial cell proliferation. In summary apocynin exhibited multifaceted pharmacological activities like nootropic, anti-oxidant, and neuroprotective in preventing the devastatingly progressive nature of Alzheimer disease. The study also urges for exploring the potential of apocynin in further in-vivo animal models to warranty its use in clinical trials.

\section{Acknowledgment}

None.

\section{Competing Interests}

The author(s) declare(s) that they have no competing interests.

\section{Funding Information}

The authors thank the UGC-BSR fellowship for the financial support.

\section{References}

1. Wouters OJ, McKee M, Luyten J (2020) Estimated research and development investment needed to bring a new medicine to market, 2009-2018. JAMA 323: 844853. [crossref]

2. Santana S, Rico EP, Burgos JS (2012) Can zebrafish be used as animal model to study Alzheimer's disease? American Journal of Neurodegenerative Disease 1.

3. Bowman TV, Zon LI (2010) Swimming into the future of drug discovery: in vivo chemical screens in zebrafish. ACS Chem Biol 19: 159-161. [crossref]

4. Rico EP, Rosemberg DB, Seibt KJ, Capiotti KM, Da Silva RS, et al. (2011) Zebrafish neurotransmitter systems as potential pharmacological and toxicological targets. Neurotoxicology and Teratology 33:608-617. [crossref]

5. Barbazuk WB, Korf I, Kadavi C, Heyen J, Tate S, et al. (2000) The syntenic relationship of the zebrafish and human genomes. Genome Research 10:1351-1358. [crossref]

6. Mussulini BH, Leite CE, Zenki KC, Moro L, Baggio S, et al. (2013) Seizures induced by pentylenetetrazole in the adult zebrafish: a detailed behavioral characterization. PloS ONE 8. [crossref]

7. Mueller T, Vernier P, Wullimann MF (2004) The adult central nervous cholinergic system of a neurogenetic model animal, the zebrafish Danio rerio. Brain Research 1011:156-69. [crossref]

8. Newman M, Verdile G, Martins RN, Lardelli M (2011) Zebrafish as a tool in Alzheimer's disease research. Biochimica et Biophysica Acta (BBA)-Molecular Basis of Disease 1812: 346-352. [crossref]
9. Schmäl F (2013) Neuronal mechanisms and the treatment of motion sickness Pharmacology 91: 229-241.

10. Golding JF, Gresty MA (2015) Pathophysiology and treatment of motion sickness. Current Opinion in Neurology 28: 83-84. [crossref]

11. Atri A, Sherman S, Norman KA, Kirchhoff BA, Nicolas MM, et al. (2004) Blockade of central cholinergic receptors impairs new learning and increases proactive interference in a word paired-associate memory task. Behavioral Neuroscience 118 [crossref]

12. Green A, Ellis KA, Ellis J, Bartholomeusz CF, Ilic S, et al. (2005) Muscarinic and nicotinic receptor modulation of object and spatial n-back working memory in humans. Pharmacology Biochemistry and Behavior 81: 575-584. [crossref]

13. Lee JC, Park JH, Ahn JH, Park J, Kim IH, et al. (2018) Effects of chronic scopolamine treatment on cognitive impairment and neurofilament expression in the mouse hippocampus. Molecular Medicine Reports 17: 1625-32. [crossref]

14. Skalicka-Wozniak K, Budzynska B, Biala G, Boguszewska-Czubara A (2018) Scopolamine-induced memory impairment is alleviated by xanthotoxin: role of acetylcholinesterase and oxidative stress processes. ACS Chemical Neuroscience 9:1184-1194. [crossref]

15. Kim YH, Lee Y, Kim D, Jung MW, Lee CJ (2010) Scopolamine-induced learning impairment reversed by physostigmine in zebrafish. Neuroscience Research 67:156161. [crossref]

16. Richetti SK, Blank M, Capiotti KM, Piato AL, Bogo MR, et al. (2011) Quercetin and rutin prevent scopolamine-induced memory impairment in zebrafish. Behavioural Brain Research 217: 10-15. [crossref]

17. Cognato GD, Bortolotto JW, Blazina AR, Christoff RR, Lara DR, et al. (2012) Y-Maze memory task in zebrafish (Danio rerio): the role of glutamatergic and cholinergic systems on the acquisition and consolidation periods. Neurobiology of Learning and Memory 98: 321-328. [crossref]

18. Bortolotto JW, de Melo GM, de Paula Cognato G, Vianna MR, Bonan CD. (2015) Modulation of adenosine signaling prevents scopolamine-induced cognitive impairment in zebrafish. Neurobiology of Learning and Memory 118: 113-119.

19. Rajesh V, Ilanthalir S (2016) Cognition enhancing activity of sulforaphane against scopolamine induced cognitive impairment in zebra fish (Danio rerio). Neurochemical Research 41: 2538-2548. [crossref]

20. Zanandrea R, Abreu MS, Piato A, Barcellos LJ, Giacomini AC. (2018) Lithium prevents scopolamine-induced memory impairment in zebrafish. Neuroscience Letters 18: 664: 34-37.

21. Stefanska J, Pawliczak R. (2008) Apocynin: molecular aptitudes. Mediators of Inflammation. [crossref]

22. Romanini CV, Ferreira ED, Soares LM, Santiago AN, Milani H, et al. (2015) 4hydroxy-3-methoxy-acetophenone-mediated long-lasting memory recovery, hippocampal neuroprotection, and reduction of glial cell activation after transient global cerebral ischemia in rats. Journal of Neuroscience Research 93:1240-1249. [crossref]

23. t Hart BA, Copray S, Philippens I (2014) Apocynin, a low molecular oral treatment for neurodegenerative disease. BioMed Research International. [crossref]

24. Engels F, Renirie BF, 'Hart BA, Labadie RP, Niikamp FP (1992) Effects of apocynin, a drug isolated from the roots of Picrorhiza kurroa, on arachidonic acid metabolism. FEBS Letters 305: 254-256. [crossref]

25. Philippens IH, Wubben JA, Finsen B, A't Hart B (2013) Oral treatment with the NADPH oxidase antagonist apocynin mitigates clinical and pathological features of parkinsonism in the MPTP marmoset model. Journal of Neuroimmune Pharmacology 8: 715-726. [crossref]

26. OECD (2019), Test No. 203: Fish, Acute Toxicity Test, OECD Guidelines for the Testing of Chemicals, Section 2, OECD Publishing, Paris. https://doi. org/10.1787/9789264069961-en

27. Braida D, Ponzoni L, Martucci R, Sparatore F, Gotti C, Sala M (2014) Role of neuronal nicotinic acetylcholine receptors (nAChRs) on learning and memory in zebrafish. Psychopharmacology 231:1975-1985. [crossref]

28. Kinkel MD, Eames SC, Philipson LH, Prince VE (2010) Intraperitoneal injection into adult zebrafish. JoVE (Journal of Visualized Experiments). [crossref]

29. Collymore C, Rasmussen S, Tolwani RJ (2013) Gavaging adult zebrafish. Journal of 
visualized experiments: JoVE.

30. Manuel R, Gorissen M, Piza Roca C, Zethof J, Vis HV, et al. (2014) Inhibitory avoidance learning in zebrafish (Danio rerio): effects of shock intensity and unraveling differences in task performance. Zebrafish 11: 341-352. [crossref]

31. Pereira AG, Jaramillo ML, Remor AP, Latini A, Davico CE, et al. (2018) Lowconcentration exposure to glyphosate-based herbicide modulates the complexes of the mitochondrial respiratory chain and induces mitochondrial hyperpolarization in the Danio rerio brain. Chemosphere 209: 353-362. [crossref]

32. Kwon SH, Lee HK, Kim JA, Hong SI, Kim HC, Jo TH, Park YI, Lee CK, Kim YB, Lee SY, Jang CG. (2010) Neuroprotective effects of chlorogenic acid on scopolamineinduced amnesia via anti-acetylcholinesterase and anti-oxidative activities in mice. European Journal of Pharmacology 649: 210-217. [crossref]

33. Ghumatkar PJ, Patil SP, Jain PD, Tambe RM, Sathaye S (2015) Nootropic, neuroprotective and neurotrophic effects of phloretin in scopolamine induced amnesia in mice. Pharmacology Biochemistry and Behavior 135: 182-191. [crossref]

34. Falsafi SK, Deli A, Höger H, Pollak A, Lubec G (2012) Scopolamine administration modulates muscarinic, nicotinic and NMDA receptor systems. PloS ONE 7. [crossref]

35. Yang W, Yu J, Zhao L, Ma N, Fang Y, et al. (2015) Polysaccharides from Flammulina velutipes improve scopolamine-induced impairment of learning and memory of rats.
Journal of Functional Foods 18: 411-422.

36. Kim MS, Lee DY, Lee J, Kim HW, Sung SH, et al. (2018) Terminalia chebula extract prevents scopolamine-induced amnesia via cholinergic modulation and antioxidative effects in mice. BMC Complementary and Alternative Medicine 18:1-1. [crossref]

37. Budzynska B, Boguszewska-Czubara A, Kruk-Slomka M, Skalicka-Wozniak K Michalak A, Musik I, Biala G. (2015) Effects of imperatorin on scopolamine-induced cognitive impairment and oxidative stress in mice. Psychopharmacology 232: 931942. [crossref]

38. Todirascu-Ciornea E, El-Nashar HA, Mostafa NM, Eldahshan OA, Boiangiu RS, et al. (2019) Schinus terebinthifolius essential oil attenuates scopolamine-induced memory deficits via cholinergic modulation and antioxidant properties in a zebrafish model. Evidence-Based Complementary and Alternative Medicine.

39. Sun AY, Wang Q, Simonyi A, Sun GY (2008) Botanical phenolics and brain health. Neuromolecular Medicine 10: 259-274.

40. Jha MK, Jeon S, Suk K (2012) Glia as a link between neuroinflammation and neuropathic pain. Immune Network 12: 41-47. [crossref]

\section{Citation:}

Bagle S, Muke S, Peshattiwar V, Kaikini A, Sathaye S (2021) Nootropic, Neuroprotective and Anti-oxidant Role of Apocynin in Scopolamine Induced Memory Deficit in a Zebrafish Model. J Pharmacol Pharm Res Volume 4(1): 1-8. 